\title{
ON INVERTING THE KOSZUL COMPLEX
}

\author{
KAMAL KHURI-MAKDISI
}

\begin{abstract}
Let $V$ be an $n$-dimensional vector space. We construct an exact sequence that gives a $G L(V)$-equivariant "resolution" of each symmetric power $\mathrm{S}^{t} V$ in terms of direct sums of tensor products of the form $\wedge^{i_{1}} V \otimes \cdots \otimes \wedge{ }^{i} p V$. This exact sequence corresponds to inverting the relation in the representation ring of $G L(V)$ that is described by the Koszul complex.
\end{abstract}

Let $k$ be a field, and let $V$ be an $n$-dimensional vector space over $k$ with $n \geq 2$. We view the alternating and symmetric powers $\wedge^{i} V$ and $S^{t} V$ of $V$ as representations of $G=G L(V) \cong G L(n, k)$; we allow any $i, t \in \mathbf{Z}$, with the understanding that $\wedge^{i} V$ and $S^{t} V$ are zero unless $0 \leq i \leq n$ and $t \geq 0$. Working in the representation ring of $G$ (i.e., the Grothendieck group of algebraic representations of $G$ ), we can write $S^{t} V$ as a polynomial in the fundamental representations $V=\wedge^{1} V, \wedge^{2} V, \ldots, \wedge^{n} V$; as we shall see below, the terms of this polynomial can be ordered in a natural way with alternating signs. In this note, we show that it is possible to concretely realize this polynomial expression for $S^{t} V$ by an exact sequence of representations of $G$. Our result thus generalizes the result of KM03 from $S L(2)$ to $G L(n)$.

The first few cases of our exact sequence, for $1 \leq t \leq 4$, are

$$
\begin{aligned}
0 \rightarrow & \rightarrow S^{1} V \rightarrow 0, \quad 0 \rightarrow \wedge^{2} V \rightarrow V \otimes V \rightarrow S^{2} V \rightarrow 0, \\
0 \rightarrow & \wedge^{3} V \rightarrow\left(V \otimes \wedge^{2} V\right) \oplus\left(\wedge^{2} V \otimes V\right) \rightarrow V \otimes V \otimes V \rightarrow S^{3} V \rightarrow 0, \\
0 \rightarrow & \wedge^{4} V \rightarrow\left(V \otimes \wedge^{3} V\right) \oplus\left(\wedge^{2} V \otimes \wedge^{2} V\right) \oplus\left(\wedge^{3} V \otimes V\right) \rightarrow \\
& \rightarrow\left(V \otimes V \otimes \wedge^{2} V\right) \oplus\left(V \otimes \wedge^{2} V \otimes V\right) \oplus\left(\wedge^{2} V \otimes V \otimes V\right) \rightarrow \\
& \rightarrow V \otimes V \otimes V \otimes V \rightarrow S^{4} V \rightarrow 0 .
\end{aligned}
$$

In general, the sequence has the form

$$
0 \rightarrow T_{t}^{1} \stackrel{\delta}{\rightarrow} T_{t}^{2} \stackrel{\delta}{\rightarrow} \cdots \stackrel{\delta}{\rightarrow} T_{t}^{t} \stackrel{\delta}{\rightarrow} S^{t} V \rightarrow 0
$$

for suitable differentials $\delta$, where, for $1 \leq p \leq t$, the $p$ th term $T_{t}^{p}$ is given by

$$
\begin{aligned}
T_{t}^{p} & =\bigoplus_{\substack{1 \leq i_{1}, \ldots, i_{p} \leq n \\
i_{1}+\cdots+i_{p}=t}}\left(\wedge^{i_{1}} V \otimes \cdots \otimes \wedge^{i_{p}} V\right) \\
& \cong \bigoplus_{\substack{\ell_{1}, \ldots, \ell_{n} \geq 0 \\
\ell_{1}+2 \ell_{2}+\cdots+n \\
\ell_{1}+\ell_{2}+\cdots+\ell_{n}=p}} \frac{\left(\ell_{1}+\cdots+\ell_{n}\right) !}{\ell_{1} ! \cdots \ell_{n} !}\left[V^{\otimes \ell_{1}} \otimes\left(\wedge^{2} V\right)^{\otimes \ell_{2}} \cdots \otimes\left(\wedge^{n} V\right)^{\otimes \ell_{n}}\right] .
\end{aligned}
$$

In particular, $T_{t}^{1}=\wedge^{t} V$, which may be zero (more generally, the condition $i_{1}, \ldots, i_{p} \leq$ $n$ in (3) is redundant since otherwise $\left.\wedge^{i} V=0\right)$, and $T_{t}^{t}=V \otimes \cdots \otimes V(t$ times $)$. Our

2000 Mathematics Subject Classification. 20G05, 15A72, 16E05.

February 27, 2007. 
choice of letters $t$ and $p$ refers to the "total degree" (i.e., the effect of a scalar matrix in $G$ ) and "partial degree" (i.e., the number of parts) of a decomposable tensor $\alpha_{1} \otimes \cdots \otimes \alpha_{p} \in \wedge^{i_{1}} V \otimes \cdots \otimes \wedge^{i_{p}} V \subset T_{t}^{p}$. The multinomial coefficient $\frac{\left(\ell_{1}+\cdots+\ell_{n}\right) !}{\ell_{1} ! \cdots \ell_{n} !}$ refers to a direct sum of several copies of the representation $V^{\otimes \ell_{1}} \otimes \cdots \otimes \wedge^{n} V^{\otimes \ell_{n}}$ of $G$. In the special case $n=2$, we see that $T_{t}^{t-i} \cong\left(\begin{array}{c}t-i \\ i\end{array}\right)\left[V^{\otimes(t-2 i)} \otimes\left(\wedge^{2} V\right)^{\otimes i}\right]$, and we recover the result of [KM03].

Before describing the differentials in (2), we pause to explain why

$$
T_{t}^{1}-T_{t}^{2}+\cdots+(-1)^{t-1} T_{t}^{t}+(-1)^{t} S^{t} V=0
$$

in the representation ring of $G$. This gives the polynomial expression of $S^{t} V$ in terms of the $\wedge^{i} V$, and arises from inverting the relation between symmetric and alternating powers that is expressed by the exactness of the Koszul complex

$$
0 \rightarrow \wedge^{n} V \otimes S^{*} V \rightarrow \cdots \rightarrow \wedge^{2} V \otimes S^{*} V \rightarrow V \otimes S^{*} V \rightarrow S^{*} V \rightarrow k \rightarrow 0 .
$$

Here $S^{*} V=\oplus_{t>0} S^{t} V$ is the symmetric algebra on $V$; it is naturally isomorphic to the polynomial algebra $k\left[e_{1}, \ldots, e_{n}\right]$, with $\left\{e_{1}, \ldots, e_{n}\right\}$ a basis for $V$. The last term $k$ is the trivial representation of $G$, i.e., the unit element of the representation ring of $G$; this term should be viewed as $k\left[e_{1}, \ldots, e_{n}\right] /\left\langle e_{1}, \ldots, e_{n}\right\rangle$.

The morphisms in (5) increase the degree in each $S^{*} V$ component by one, so by taking $G$-equivariant Hilbert series with a formal parameter $x$, we obtain the identity of formal power series in the representation ring of $G$ :

$$
\left(\sum_{t=0}^{\infty} S^{t} V \cdot x^{t}\right)\left(1-V \cdot x+\wedge^{2} V \cdot x^{2}-\cdots+(-1)^{n} \wedge^{n} V \cdot x^{n}\right)=1 .
$$

Now replace $x$ by $-x$ and invert the sum over the $\wedge^{i} V$ to yield

$$
\begin{aligned}
\sum_{t=0}^{\infty}(-1)^{t} S^{t} V \cdot x^{t} & =\frac{1}{1+V \cdot x+\wedge^{2} V \cdot x^{2}+\cdots+\wedge^{n} V \cdot x^{n}} \\
& =\sum_{p=0}^{\infty}(-1)^{p}\left(V \cdot x+\wedge^{2} V \cdot x^{2}+\cdots+\wedge^{n} V \cdot x^{n}\right)^{p} .
\end{aligned}
$$

For $t \geq 1$, the coefficient of $x^{t}$ in $\left(V \cdot x+\wedge^{2} V \cdot x^{2}+\cdots+\wedge^{n} V \cdot x^{n}\right)^{p}$ is zero unless $1 \leq p \leq t$, in which case this coefficient is the class of $T_{t}^{p}$. This proves (4).

We now define the differential $\delta$ of (2) on each direct summand $\wedge^{i_{1}} V \otimes \cdots \otimes \wedge^{i_{p}} V$ of $T_{t}^{p}$, where $t=i_{1}+\cdots+i_{p}$. We start with the case $p=1$. Given $v_{1}, \ldots, v_{i} \in V$, we introduce the notations $v_{A}$, for nonempty $A \subset\{1, \ldots, i\}$, and $s(A, B)$, for disjoint nonempty $A, B \subset\{1, \ldots, i\}$, by

$$
\begin{gathered}
v_{A}=v_{a_{1}} \wedge \cdots \wedge v_{a_{j}} \in \wedge^{j} V, \text { where } A=\left\{a_{1}, \ldots, a_{j}\right\} \text { with } a_{1}<\cdots<a_{j}, \\
s(A, B) \in\{ \pm 1\} \text { such that } v_{A \cup B}=s(A, B) v_{A} \wedge v_{B} .
\end{gathered}
$$

Note that $s(A, B)$ depends only on the sets $A$ and $B$, and not on the choice of $v_{1}, \ldots, v_{i}$. We then define, for a decomposable tensor $v_{1} \wedge \cdots \wedge v_{i} \in \wedge^{i} V=T_{i}^{1}$,

$$
\delta\left(v_{1} \wedge \cdots \wedge v_{i}\right)=\sum_{\substack{\emptyset \neq A, B \subset\{1, \ldots, i\} \\ A \cup B=\{1, \ldots, i\} \\ A \cap B=\emptyset}} s(A, B) v_{A} \otimes v_{B} \in T_{i}^{2} .
$$


For example, $\delta\left(v_{1} \wedge v_{2}\right)=v_{1} \otimes v_{2}-v_{2} \otimes v_{1}$, and $\delta\left(v_{1} \wedge v_{2} \wedge v_{3} \wedge v_{4}\right)$ is

$$
\begin{aligned}
& \left(v_{1} \wedge v_{2} \wedge v_{3}\right) \otimes v_{4}-\left(v_{1} \wedge v_{2} \wedge v_{4}\right) \otimes v_{3}+\left(v_{1} \wedge v_{3} \wedge v_{4}\right) \otimes v_{2}-\left(v_{2} \wedge v_{3} \wedge v_{4}\right) \otimes v_{1} \\
& +\left(v_{1} \wedge v_{2}\right) \otimes\left(v_{3} \wedge v_{4}\right)-\left(v_{1} \wedge v_{3}\right) \otimes\left(v_{2} \wedge v_{4}\right)+[3 \text { more terms }]+\left(v_{3} \wedge v_{4}\right) \otimes\left(v_{1} \wedge v_{2}\right) \\
& +v_{1} \otimes\left(v_{2} \wedge v_{3} \wedge v_{4}\right)-v_{2} \otimes\left(v_{1} \wedge v_{3} \wedge v_{4}\right)+v_{3} \otimes\left(v_{1} \wedge v_{2} \wedge v_{4}\right)-v_{4} \otimes\left(v_{1} \wedge v_{2} \wedge v_{3}\right) .
\end{aligned}
$$

We claim that $\delta$ is well defined, i.e., that the right hand side of (9) is an alternating form in the vectors $v_{1}, \ldots, v_{i}$. The easiest way to verify this is to check that if two adjacent vectors $v_{\ell}, v_{\ell+1}$ are equal, then the right hand side of (9) vanishes. Then we define the general action of $\delta$ on $\alpha_{1} \otimes \cdots \otimes \alpha_{p} \in \wedge^{i_{1}} V \otimes \cdots \otimes \wedge^{i_{p}} V \subset T_{t}^{p}$ by

$$
\begin{aligned}
\delta\left(\alpha_{1} \otimes \cdots \otimes \alpha_{p}\right) & =\delta\left(\alpha_{1}\right) \otimes \alpha_{2} \otimes \cdots \otimes \alpha_{p} \quad-\quad \alpha_{1} \otimes \delta\left(\alpha_{2}\right) \otimes \cdots \otimes \alpha_{p} \\
& +\cdots+(-1)^{p-1} \alpha_{1} \otimes \alpha_{2} \otimes \cdots \otimes \delta\left(\alpha_{p}\right) \in T_{t}^{p+1} .
\end{aligned}
$$

Finally, the last differential $\delta: T_{t}^{t} \rightarrow S^{t} V$ is the natural projection from $T_{t}^{t}=V^{\otimes t}$.

Lemma 1. The differentials $\delta: T_{t}^{p} \rightarrow T_{t}^{p+1}$ as defined above are $G$-homomorphisms that satisfy $\delta \delta=0$. Thus (2) is a complex of $G$-representations.

Proof. From the definition of $\delta\left(v_{1} \wedge \cdots \wedge v_{i}\right)$, we see that it respects the $G$-action. (This would have been less transparent if we had defined $\delta$ from the beginning in terms of basis elements $e_{a_{1}} \wedge \cdots \wedge e_{a_{i}}$ of $\wedge^{i} V$, for a fixed basis $\left\{e_{1}, \ldots, e_{n}\right\}$ of $V$.) This implies the $G$-linearity in general. As for $\delta \delta=0$, one first checks directly that $\delta \delta\left(v_{1} \wedge \cdots \wedge v_{i}\right)=0$. The crucial ingredient is that if $A, B$, and $C$ are disjoint nonempty subsets of $\{1, \ldots, i\}$, then $s(A \cup B, C) s(A, B)=s(A, B \cup C) s(B, C)$. This follows from comparing $v_{A} \wedge v_{B} \wedge v_{C}$ with $v_{A \cup B \cup C}$. This settles the case $p=1$, and we then proceed inductively for larger $p$. In particular, writing $\beta=\alpha_{2} \otimes \cdots \otimes \alpha_{p}$, we have $\delta \delta(\alpha \otimes \beta)=\delta[\delta(\alpha) \otimes \beta-\alpha \otimes \delta(\beta)]$, and the reader should be careful to note the + sign in expanding the first term: $\delta[\delta(\alpha) \otimes \beta]=\delta(\delta(\alpha)) \otimes \beta+\delta(\alpha) \otimes \delta(\beta)$, because $\delta(\alpha)$ has partial degree 2. This proves our result except at the last step, involving $T_{t}^{t} \rightarrow S^{t} V$, where one can show directly that $\delta \delta=0$.

Theorem 2. The complex (2) is exact if $t \geq 1$.

Proof. It is more convenient to show instead that the truncated complex

$$
0 \rightarrow T_{t}^{1} \stackrel{\delta}{\rightarrow} T_{t}^{2} \stackrel{\delta}{\rightarrow} \cdots \stackrel{\delta}{\rightarrow} T_{t}^{t} \rightarrow 0
$$

has zero cohomology everywhere except at the last term, where the cohomology is $S^{t} V$. The proof is by induction on $t$, the case $t=1$ being trivial. For the inductive step, we introduce a filtration on the complex (12), and compute a spectral sequence. We define $\operatorname{Fil}^{f}\left(T_{t}^{p}\right) \subset T_{t}^{p}$ by

$$
\operatorname{Fil}^{f}\left(T_{t}^{p}\right)=\bigoplus_{\substack{i_{1} \leq f \\ 1 \leq i_{1}, \ldots, i_{p} \leq n \\ i_{1}+\cdots+i_{p}=t}}\left(\wedge^{i_{1}} V \otimes \cdots \otimes \wedge^{i_{p}} V\right) .
$$

(Thus $f$ is a bound on the degree $i_{1}$ of the "first term" $\alpha_{1}$ in any tensor $\alpha_{1} \otimes \cdots \otimes \alpha_{p} \in$ Fil $^{f}\left(T_{t}^{p}\right)$.) We have $T_{t}^{p}=\operatorname{Fil}^{t}\left(T_{t}^{p}\right) \supset \cdots \supset \operatorname{Fil}^{0}\left(T_{t}^{p}\right)=0$. The $f$ th graded part of the complex, for $1 \leq f \leq t$, is $_{\operatorname{Gr}^{f}}\left(T_{t}^{p}\right)=\operatorname{Fil}^{f}\left(T_{t}^{p}\right) / \mathrm{Fil}^{f-1}\left(T_{t}^{p}\right)$, and

$$
\operatorname{Gr}^{f}\left(T_{t}^{p}\right) \cong \bigoplus_{\substack{i_{1}=f \\ i_{1}+\cdots+i_{p}=t}}\left(\wedge^{i_{1}} V \otimes \cdots \otimes \wedge^{i_{p}} V\right) \cong \wedge^{f} V \otimes T_{t-f}^{p-1} .
$$


Here we slightly abuse notation, since $\operatorname{Gr}^{t}\left(T_{t}^{1}\right)=\wedge^{t} V$, corresponding to taking $T_{0}^{0}=k$ in the exceptional case when we simultaneously have $p=1$ and $f=t$; if $p=1$ and $f \neq t$, we take $T_{t-f}^{0}$ and $\operatorname{Gr}^{f}\left(T_{t}^{1}\right)$ to be zero, consistently with (3). Now $\delta$ respects the filtration, and descends to a differential $\bar{\delta}: \operatorname{Gr}^{f}\left(T_{t}^{p}\right) \rightarrow \operatorname{Gr}^{f}\left(T_{t}^{p+1}\right)$ that can be identified with $1 \otimes(-\delta): \wedge^{f} V \otimes T_{t-f}^{p-1} \rightarrow \wedge^{f} V \otimes T_{t-f}^{p}$. Applying our inductive hypothesis, and noting that the presence of $-\delta$ instead of $\delta$ makes no difference, we see that the $\bar{\delta}$-cohomology of $\operatorname{Gr}^{f}\left(T_{t}^{\bullet}\right)$ is concentrated in degree $\bullet=t-f+1$, where it is naturally isomorphic to $\wedge^{f} V \otimes S^{t-f} V$ (this holds even if $f=t$ ). Hence the $E_{1}$ term of our spectral sequence is

$$
0 \rightarrow \wedge^{t} V \otimes S^{0} V \rightarrow \wedge^{t-1} V \otimes S^{1} V \rightarrow \cdots \rightarrow \wedge^{1} V \otimes S^{t-1} V \rightarrow 0,
$$

with differentials induced from the "portion" of the original differentials $\delta$ on

$$
0 \rightarrow \wedge^{t} V \rightarrow \wedge^{t-1} V \otimes V \rightarrow \wedge^{t-2} V \otimes V \otimes V \rightarrow \cdots \rightarrow \wedge^{1} V \otimes V^{\otimes(t-1)} \rightarrow 0 .
$$

Note that (16) is not a complex, as the composition of the "partial" $\delta$ s is not zero, but the induced maps in (15) do give a complex - in fact, (15) is the part of the Koszul complex (5) in total degree $t$, after we drop the final terms $S^{*} V \rightarrow k \rightarrow 0$. (The fact that the Koszul complex, as written, starts with $\wedge^{n} V$, whereas we have started with $\wedge^{t} V$ is immaterial, since the "missing" vector spaces are all zero, either from $\wedge^{i} V$ with $i>n$, or from $S^{j} V$ with $j<0$.) Since the term $k$ in the Koszul complex only appears in total degree 0 , we obtain that the $E_{2}$ term of our spectral sequence is degenerate, consisting of a single instance of $S^{t} V$ in one corner. Thus the complex (12) has the expected cohomology.

We include a diagram of our filtered complex below in the case $t=4$, drawn so that we obtain a second quadrant spectral sequence. The partial degree $p$ of our complex is constant on SW-NE diagonals. Note that this is not a double complex, due to the diagonal arrows. The filtration corresponds to taking all columns to the right of some vertical line, and the graded complex $\operatorname{Gr}^{f}\left(T_{t}^{\bullet}\right)$ only sees the vertical arrows. Only the bottom row survives into $E_{1}$, to become (15). The $E_{2}$ term consists of just $S^{4} V$ in the SE corner.

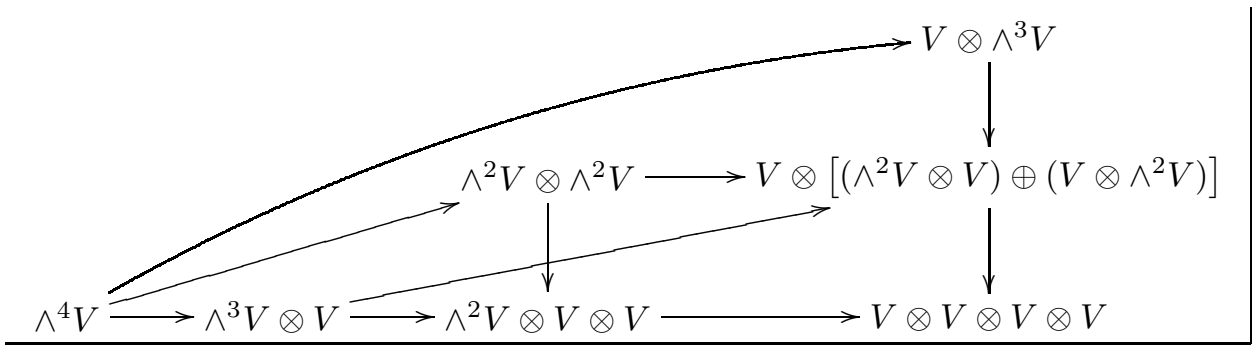

\section{REFERENCES}

[KM03] Kamal Khuri-Makdisi, An exact sequence in the representation theory of SL(2), Comm. Algebra 31 (2003), no. 9, 4153-4160. MR 1995526 (2004f:20087)

Mathematics Department and Center for Advanced Mathematical Sciences, American University of Beirut, Bliss Street, Beirut, Lebanon

E-mail address: kmakdisi@aub.edu.lb 